\title{
Countering method for active jamming based on dual-polarization radar seeker
}

\author{
QIANG ZHANG AND WEIJUN PAN
}

\begin{abstract}
In order to counter active jamming, an adaptive polarization cancellation method based on dual-polarization radar is proposed in the paper. First, respectively being obtained by vertical and horizontal polarization channels of dual-polarization radar, the signals from the real target and active jamming are analyzed in composition and characteristic. Then, the weight coefficient is timely derived in practical scene to adjust difference of amplitude and phase of active jamming signals respectively acquired by the two orthogonal polarization channels, so the method can completely eliminate active jamming, but just a little power change for the real target signal. Furthermore, a compensating method for the target signal is developed to improve the angle measuring accuracy of monopulse radars. And the influence factors of adaptive polarization cancellation are analyzed in detail by simulation. Finally, the experimental results prove that it is very effective on both active blanket jamming and active deception jamming.
\end{abstract}

Keywords: Radar signal processing and system modeling, Radar applications, Polarization technology

Received 26 February 2016; Revised 30 October 2016; Accepted 31 October 2016; first published online 29 November 2016

\section{INTRDDUCTIDN}

As a significant electromagnetic interference, active jamming is a great threat to the survival of guidance radars. It can be classified into active blanket jamming and active deception jamming. By active blanket jamming is meant the suppression noise with high power to reduce signal-to-noise ratio (SNR) of the received signal, while by active deception jamming is meant the electromagnetic decoy as the same characteristic as the real target in time-frequency domain. In order to interfere in various radars with different polarization forms, the two types of active jamming always take the form of circular polarization $[1-3]$.

Since for the difference between active jamming and the target in polarization field, the application of polarization technology for countering active jamming is drawing more and more scholars' attention. The main methods for countering active jamming are polarization encoding [4, 5], hypercomplex and spatiotemporal-polarization processing [6], and polarization filtering [7-9]. The first two methods are difficult to apply in practical engineering, because they need complex systems and not meet the requirement of miniaturization. While which is studied more extensively, the method of polarization filter can form single-notch or multinotch at the polarization angle of jamming through polarization diversity. So the radar can receive normal target echo, but less electromagnetic wave signal from active jamming,

College of Air Traffic Management, Civil Aviation Flight University of China, Guanghan 618307, China. Phone: +8608385182541

Corresponding author:

Q. Zhang

Email: zhangqiang_ioe@126.com certainly the SNR of the received signal is greatly improved. But an additional bulky polarization transformation device must be utilized to make the polarization form of the radar against that of the present active jamming. A null phase-shift polarization filtering method was presented by Mao and Liu [10]. Adaptively adjusting the parameters of polarization filter according to the polarization form of jamming and compensating the error of phase and amplitude after filtering, it can reduce the error between the filtered signal and the real target signal, and not disturb coherent processing of the filtered signal. But it is also difficult to meet the requirements of radar seekers, because it still requires a large amount of front-end equipment to deal with the variation of polarization forms. An improved method based on the research of literature [10] was proposed [11]. The polarization parameters of echo signal are obtained just by analyzing the modulation influence of spatial polarization of the guidance radar with a single polarization antenna, and then it does the same filtering processing as literature [10]. The method seems to reduce complexity of the front-end equipment of polarization radars. But the gain of orthogonal polarization pattern for a single polarization antenna is usually $30 \mathrm{~dB}$ lower than that of main polarization pattern, evidently the reliability of the obtained polarization parameters is greatly lowered and the result of countering active jamming is also not desirable enough.

With the development of antenna technology, multipolarization antennas with common aperture $[12,13]$ are no longer a problem, which will greatly accelerate the application of polarization anti-jamming technology to guidance radars. According to the public reporting, there is a dual-polarization radar seeker at $94 \mathrm{GHz}$ carried by brimstone missile on active service, which is developed by a MBDA missile system [14]. 
A polarization ECCM technique was proposed to counter active blanket jamming based on dual-polarization radar [15]. Because of neglecting the cross-polarized component of the target echo and not being compensated, the method does poorly in angle measurement. Based on the practical engineering application of dual-polarization radar seekers, this paper puts forward an adaptive polarization cancellation method to counter active jamming. Without increasing any other hardware more, the method can achieve good cancellation effects on both active blanket jamming and active deception jamming, so it is very meaningful for engineering application.

The remainder of this paper is organized as follows. Signal models of a real target and active jamming are introduced in Section II. The characteristic of the dual-polarization radar is analyzed and the practical signals obtained by the dualpolarization radar are described in Section III. Then Section IV addresses the principle of adaptive polarization cancellation, and discusses the influence factors of cancellation result. Experimental results and analysis are shown in Section V. Finally, Section VI concludes the paper.

\section{SIGNAL MODELS DFRADAR \\ TARGET AND ACTIVE JAMMING}

In a horizontal and vertical polarization coordinate system, electromagnetic wave signal transmitted by radar can be described as

$$
\boldsymbol{e}_{\boldsymbol{t}}(\boldsymbol{t})=g \sqrt{\frac{P_{t}}{4 \pi L_{t}}} e^{j_{2} \pi f_{c} t} \boldsymbol{s}(t) \boldsymbol{h},
$$

where $g$ is the gain of radar antenna, $P_{t}$ is the peak power of transmitted signal, $L_{t}$ is the comprehensive loss, $f_{c}$ is the carrier frequency, $s(t)$ is the modulation function of radar signal, and $\boldsymbol{h}=\left[\begin{array}{ll}h_{H} & h_{V}\end{array}\right]$ is the polarization form of the antenna. For convenience of description, the transmitted signal can be simplified as

$$
\boldsymbol{e}_{\boldsymbol{t}}(\boldsymbol{t})=g A_{t} \boldsymbol{h},
$$

where $A_{t}=\sqrt{\left(P_{t} / 4 \pi L_{t}\right)} e^{j 2 \pi f_{c} t} s(t)$.

So at the receiving port of radar antenna, the backscattering echo from a target can be described as

$$
\boldsymbol{e}_{i}(\boldsymbol{t})=\frac{g}{4 \pi R^{2}} A_{t}(t-\tau) e^{j 2 \pi f_{d}(t-\tau)} \boldsymbol{h S}
$$

Here $R$ represents the slant range between the radar and the target; $f_{d}$ represents the Doppler frequency caused by target motion; $\tau=2 R / c$ represents the delay time of target echo, $c$ represents the speed of light;

$$
\boldsymbol{S}=\left[\begin{array}{ll}
S_{H H} & S_{H V} \\
S_{V H} & S_{V V}
\end{array}\right]
$$

denotes the polarization scattering matrix (PSM) of the target.

While the signal from active jamming at the receiving port of radar antenna can be described as

$$
\boldsymbol{e}_{\boldsymbol{J}}(\boldsymbol{t})=J(t) \boldsymbol{h}_{\boldsymbol{J}}
$$

where $\boldsymbol{h}_{\boldsymbol{J}}=\left[\begin{array}{ll}h_{j H} & h_{j V}\end{array}\right]$ denotes the polarization form of active jamming, which is usually circular polarization; $J(t)$ is the modulation signal of active jamming.

As is mentioned above, the practical signal received by radar consists of that from the target and that from active jamming. It can be expressed as

$$
E_{r}(t)=\frac{k_{R F} g}{L_{r}}\left[\boldsymbol{e}_{\boldsymbol{i}}(\boldsymbol{t})+\boldsymbol{e}_{J}(\boldsymbol{t})\right] \boldsymbol{h}^{\boldsymbol{T}}+n(t),
$$

where $k_{R F}$ represents the RF amplification coefficient of radar receiver, $L_{r}$ is the loss of the receiver, and $n(t)$ is the receiver noise that always obeys normal distribution.

\section{CHARACTERISTIC DF DUAL-PDLARIZATION RADAR}

Formulated by Sinclair, PSM can absolutely denote the polarization characteristic of a target, which is able to be obtained by full polarization radar [16]. It is defined as

$$
\begin{aligned}
\boldsymbol{S} & =\left[\begin{array}{ll}
S_{H H} & S_{H V} \\
S_{V H} & S_{V V}
\end{array}\right] \\
& =e^{j \omega}\left[\begin{array}{cc}
\left|S_{H H}\right| & \left|S_{H V}\right| e^{j\left(\emptyset_{H V}-\omega\right)} \\
\left|S_{V H}\right| e^{j\left(\emptyset_{V H}-\omega\right)} & \left|S_{V V}\right| e^{j\left(\emptyset_{V V}-\omega\right)}
\end{array}\right],
\end{aligned}
$$

where $S_{H V}$ denotes the vertical polarization backscattering coefficient of the target irradiated by horizontal polarization wave, $S_{H H}, S_{V H}$, and $S_{V V}$ are the same definition as $S_{H V}, \omega$ is the phase of $S_{H H}, \emptyset_{H V}, \emptyset_{V H}$, and $\emptyset_{V V}$ are respective phases of $S_{H V}, S_{V H}$, and $S_{V V}$.

In practical engineering applications, dual-polarization radars are commonly used. They have the ability of single polarization transmission, but dual-polarization channels receiving simultaneously. Taking vertical polarization transmission for example, only half PSM of the target can be obtained, and it is simplified as

$$
S=\left[\begin{array}{ll}
S_{V H} & S_{V V}
\end{array}\right]
$$

Here $S_{V V}$ represents the co-polarized component that includes the same echo information as the conventional single polarization radar; $S_{V H}$ represents the cross-polarized component, which denotes the converting polarization ability of the target and is the main polarization characteristic obtained by dual-polarization radars.

For typical radar targets, such as aircrafts, warships, tanks, buildings, and so on, the polarization form of their backscattering electromagnetic wave is greatly related to that of the incident electromagnetic wave. It means that the polarization form of their backscattering electromagnetic wave is mainly as the same as that of the incident electromagnetic wave. In other words, their co-polarized ability is much greater than the converting polarization ability. So the value of $S_{V V}$ is much greater than $S_{V H}$, and that is to say $\left|S_{T V V}\right| \gg\left|S_{T V H}\right|[17]$. Here $S_{T V V}$ and $S_{T V H}$ are respectively $S_{V V}$ and $S_{V H}$ of the targets.

For active jamming, it is usually of circular polarization. Thus, its polarization form can be expressed as $\boldsymbol{h}_{\boldsymbol{J}}=[j \cdot \sin w t$ $\cos w t]$ or $\boldsymbol{h}_{\boldsymbol{J}}=[j \cdot \sin w t \cos w t]$, which respectively denote left- and right-hand circular polarizations. The powers received by vertical- and horizontal polarization antennas 
from active jamming with standard circular polarization are equal, but they are $3 \mathrm{~dB}$ lower than that received by the matching circular polarization antenna. If active jamming is considered as a special target, it is evidently $\left|S_{J V V}\right|=\left|S_{J V H}\right|$, and the difference between $S_{J V V}$ and $S_{J V H}$ is just the phase [2]. Here $S_{J V V}$ and $S_{J V H}$ are respectively $S_{V V}$ and $S_{V H}$ of active jamming. Therefore, the method of polarization cancellation can be introduced to suppress active jamming. Note that the polarization form of active jamming in practical engineering is not usually a standard circle, so the axial ratio of the circle is just close to but not 1 . That is to say, it is usually $\left|S_{J V V}\right| \approx\left|S_{J V H}\right|$ in practical engineering.

According to Section II, the signal models for dualpolarization radars should be developed as follows. When the radar is being interfered with by active jamming, the signal received by vertical polarization channel can be described as

$$
E_{v}(t)=\frac{k_{v R F} g_{v}}{L_{v r}}\left[\boldsymbol{e}_{i}(\boldsymbol{t})+\boldsymbol{e}_{\boldsymbol{J}}(\boldsymbol{t})\right] \boldsymbol{h}_{v}^{T}+n_{v}(t)
$$

where $k_{v R F}$ is the RF amplification coefficient of vertical polarization channel, $g_{v}$ is the gain of vertical polarization antenna, $L_{v r}$ is the receiver loss of vertical polarization channel, $\boldsymbol{h}_{\boldsymbol{v}}$ is the polarization form of vertical polarization antenna, and $n_{v}(t)$ is the noise of vertical polarization channel.

Substituting equations (3) and (4) into (8), equation (9) can be obtained.

$$
\begin{aligned}
E_{v}(t)= & \frac{k_{v R F} g_{v}}{L_{v r}} \\
& {\left[\frac{g_{v}}{4 \pi R^{2}} A_{t}(t-\tau) e^{j_{2} \pi f_{d}(t-\tau)} S_{T V V}+S_{J V V} J(t)\right] } \\
& +n_{v}(t) .
\end{aligned}
$$

In the same way, the signal received by horizontal polarization channel can be expressed as

$$
\begin{aligned}
E_{h}(t)= & \frac{k_{h R F} g_{h}}{L_{h r}} \\
& {\left[\frac{g_{v}}{4 \pi R^{2}} A_{t}(t-\tau) e^{j_{2} \pi f_{d}(t-\tau)} S_{T V H}+S_{J V H} J(t)\right] } \\
& +n_{h}(t),
\end{aligned}
$$

where $k_{h R F}$ is the RF amplification coefficient of horizontal polarization channel, $g_{h}$ is the gain of horizontal polarization antenna, $L_{h r}$ is the receiver loss of horizontal polarization channel, and $n_{h}(t)$ is the noise of horizontal polarization channel.

\section{ADAPTIVEPDLARIZATIDN CANCELLATION DF ACTIVE $\checkmark A M M I N G$}

\section{A) Principle of adaptive polarization cancellation}

From equations (9) and (10), the signals obtained by verticaland horizontal channels differ in both amplitude and phase.
To make the active jamming be completely cancelled, the amplitude and phase of active jamming signals obtained by the two channels should be made consistent. So the weight coefficient of polarization cancellation can be defined as

$$
\varphi=\frac{k_{v R F} g_{v} L_{h r} S_{J V V}}{k_{h R F} g_{h} L_{v r} S_{J V H}}=\frac{k_{v R F} g_{v} L_{h r}}{k_{h R F} g_{h} L_{v r}} \alpha e^{-j w_{v h}} .
$$

Here $\alpha=\left|S_{J V V}\right| /\left|S_{J V H}\right|$, represents the axial ratio of circular polarization; $w_{v h}$ is the phase difference between $S_{J V V}$ and $S_{J V H}$.

Then, the polarization cancellation method can be described as

$$
z(t)=E_{v}(t)-\varphi E_{h}(t)
$$

Substituting equations (9) and (10) into (12), It can be developed as

$$
\begin{aligned}
z(t)= & \frac{k_{v R F} g_{v}^{2}}{4 \pi R^{2} L_{v r}} A_{t}(t-\tau) e^{j 2 \pi f_{d}(t-\tau)}\left[S_{T V V}-S_{T V H} \alpha e^{-j w_{v h}}\right] \\
& +\left[n_{v}(t)-\frac{k_{v R F} g_{v} L_{h r}}{k_{h R F} g_{h} L_{v r}} n_{h}(t) \alpha e^{-j w_{v h}}\right] .
\end{aligned}
$$

From equation (13), active jamming can be completely eliminated in theory, but the signal power received from the target is changed. It will reduce the angle-measuring accuracy of monopulse radars with sum and difference patterns of amplitude, because the method of monopulse angle measurement is sensitive for the amplitude of the target signal $[18,19]$.

In practical engineering, both amplitude difference and phase difference between vertical and horizontal channels of the dual-polarization radar caused by the hardware system can be adjusted easily as was described in prior publications $[20,21]$. So the weight coefficient of polarization cancellation can be simplified as

$$
\varphi=\frac{E_{J v}(t)}{E_{J h}(t)}=p e^{-j w_{v h}}
$$

where $E_{J v}(t)$ represents the signal of active jamming obtained by vertical channel, $E_{J h}(t)$ represents that obtained by horizontal channel, and

$$
p=\frac{k_{v R F} g_{v} L_{h r}\left|S_{J V V}\right|}{k_{h R F} g_{h} L_{v r}\left|S_{J V H}\right|}
$$

represents the amplitude ratio of the signals obtained by the two orthogonal polarization channels. When the dualpolarization radar does not emit electromagnetic wave, its received signal must be only from active jamming. So $E_{J v}(t)$ and $E_{J h}(t)$ can be easily obtained.

In equation (12), $E_{h}(t)=E_{J h}(t)+E_{T h}(t)$, where $E_{T h}(t)$ is the horizontal component of the target echo, thus it is regarded as jamming signal and eliminated in error. To improve angle-measuring accuracy, the target signal power 
after polarization cancellation should be adjusted as

$$
\begin{aligned}
z(t) & =E_{v}(t)-\varphi E_{h}(t)+\varphi\left(E_{h}(t)-E_{J h}(t)\right) \\
& =E_{v}(t)-E_{J v}(t) .
\end{aligned}
$$

For monopulse radars, equation (15) must be used in both sum signal and difference signal simultaneously. Eliminating most active jamming signal and adjusting the power change introduced by polarization cancellation, the method will greatly improve the angle-measuring accuracy. But $E_{v}(t)$ and $E_{J v}(t)$ are not obtained at the same time, so it may introduce extra phase difference and the target signal after it cannot retain coherent perfectly.

Therefore, equations (12) and (13) should be chosen to counter active jamming when the radar needs coherent integration. Because the same value of $\varphi$ is used in the whole integration period, the phase of target signal will keep consistent and the polarization cancellation method will not reduce the gain of coherent integration. In addition, the co-polarized component is much greater than the cross-polarized component for typical radar targets and the axial ratio of active jamming is usually close to 1 , so the signal power after polarization cancellation received from the target is just made a little change. It has little influence for subsequent signal processing besides monopulse angle measurement.

The proposed method above needs not any parameters of active jamming in prior, and can adaptively obtain the weight coefficient of polarization cancellation in time. Then, it adaptively does different cancellation processing according to requirements, equation (12) for coherent integration and equation (15) for monopulse angle measurement.

\section{B) Influence factors of polarization cancellation}

According to equation (12), the weight coefficient $\varphi$ is unquestionably a key factor related to the efficiency of polarization cancellation. In the process of adaptive polarization cancellation, $\varphi$ is obtained from the actual environment in real time, then it will be used for the following echo pulses. So there are some errors between the obtained $\varphi$ and the real $\varphi$ of every pulse, certainly the loss of polarization cancellation will be introduced.

In this paper, the gain of signal-to-interference-and-noise ratio $\left(G_{\text {SINR }}\right)$ is defined as the improvement factor to evaluate the efficiency of polarization cancellation. It is expressed as

$$
G_{S I N R}=S I N R_{\text {out }}-S I N R_{\text {in }},
$$

where $S I N R_{\text {in }}$ represents signal-to-interference-and-noise ratio (SINR) of input signal, and SINR ${ }_{\text {out }}$ represents SINR of output signal. In addition, SINR is defined as

$$
\operatorname{SINR}=10 \log \frac{P_{S}}{P_{I}+P_{N}},
$$

where $P_{S}$ represents the power of the target signal, $P_{I}$ represents that of interference, and $P_{N}$ represents that of noise.

When SINRs of two input signals are -7 and $-3 \mathrm{~dB}$, their three-dimensional diagrams of $G_{S I N R}$ after polarization cancellation with the change of amplitude and phase errors of $\varphi$ are respectively shown in Figs 1 and 2. Here, the $x$-axis

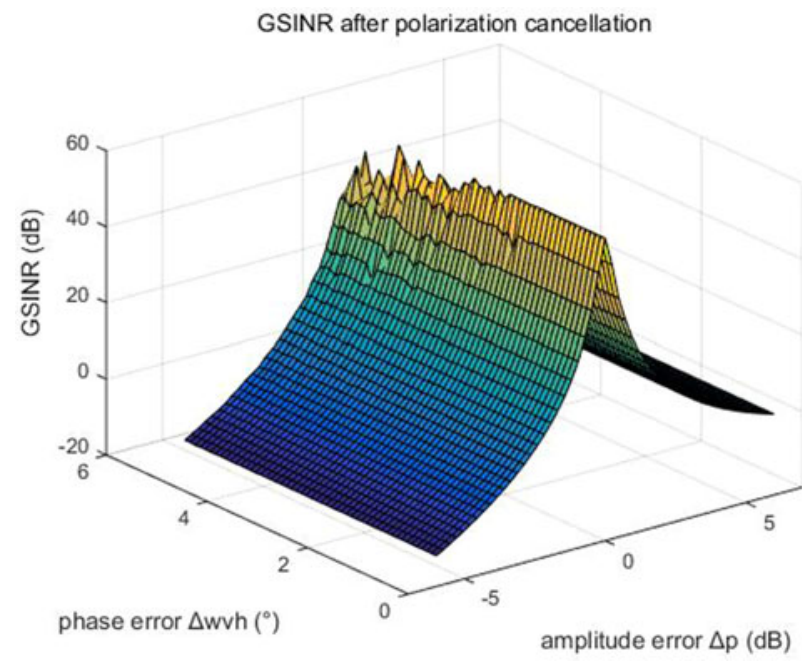

Fig. 1. $G_{S I N R}$ with the change of amplitude and phase errors of $\varphi$, when SINR $=-7 \mathrm{~dB}$.

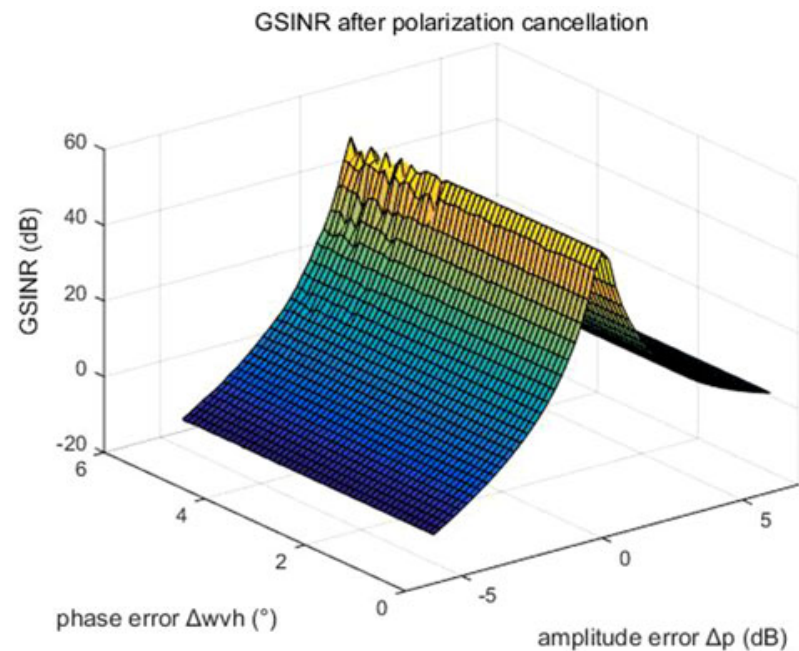

Fig. 2. $G_{S I N R}$ with the change of amplitude and phase errors of $\varphi$, when $\operatorname{SINR}=-3 \mathrm{~dB}$.

denotes the amplitude error $\Delta p$, the $y$-axis denotes the phase error $\Delta w_{v h}$, and the $z$-axis denotes $G_{S I N R}$. It is evidently that $G_{S I N R}$ is extremely sensitive to $\Delta p$ and decays rapidly with the increase of $\Delta p$, but not sensitive to $\Delta w_{v h}$.

On the other hand, noise and interference are surely influence factors of polarization cancellation. Thus, the influence of SINR is also analyzed in Fig. 3 , where the $x$-axis represents SINR of the input signal, and the $y$-axis represents $G_{\text {SINR }}$. The diagram shows that $G_{S I N R}$ increases with the increase of SINR, and $G_{\text {SINR }}$ reaches the peak when SINR is approximately $10 \mathrm{~dB}$, then decays slowly. Note that SINR most depends on the radar system and the practical scene, so it is difficult to be improved.

In the two simulations, the radar signal is linear frequency modulation signal, and the ratio of co-polarized-to-crosspolarized component of the target signal is 6 . According to the analysis above, it is obviously that the amplitude value of $\varphi$ is the most sensitive factor, so ensuring real time update of $\varphi$ and reducing its error is very important in the process of polarization cancellation. 


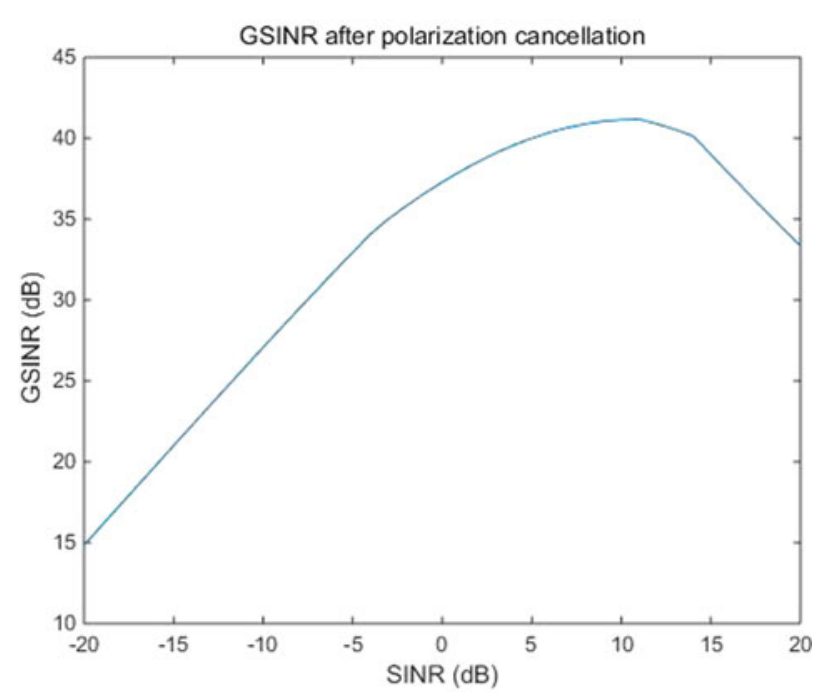

Fig. 3. $G_{S I N R}$ with the change of SINR.

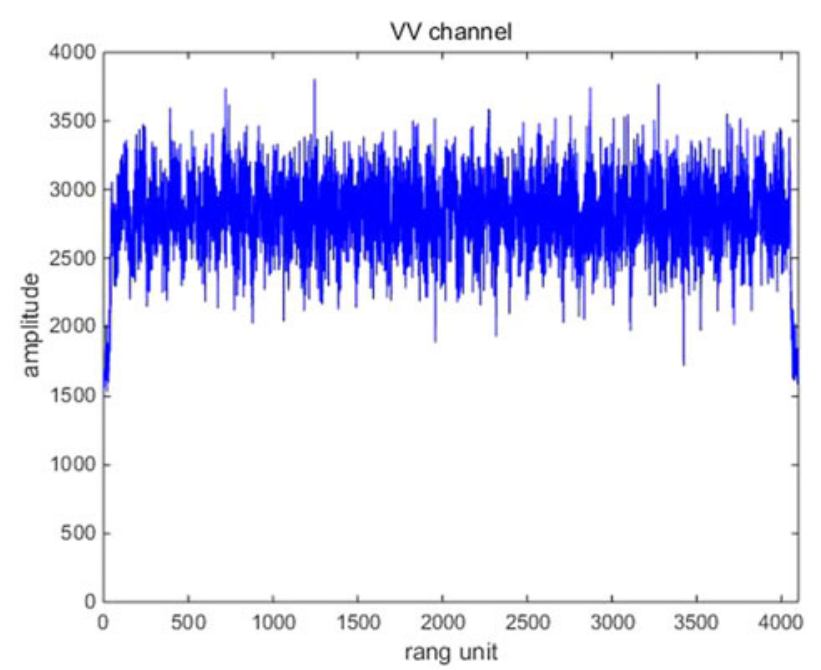

Fig. 4. Range profile of the VV channel for active blanket jamming.

\section{EXPERIMENTAL RESULTS AND ANALYSIS}

In order to prove the effectiveness of the proposed method for countering active jamming, real experimental data obtained by the dual-polarization radar in millimeter wave band is analyzed. In the experiment, an angle reflector is used as the target, and it is approximately 1000 range units far from the radar, then an active blanket jammer and an active deception jammer are respectively used to emit interference signal at the radar.

Their results after the proposed adaptive polarization cancellation for active blanket jamming are shown in Figs 4-6. In which, Fig. 4 is the one-dimensional range profile obtained by vertical polarization transmission and vertical polarization receiving (VV) channel of the dual-polarization radar, Fig. 5 is that obtained by vertical polarization transmission and horizontal polarization receiving $(\mathrm{VH})$ channel, and Fig. 6 is the result after polarization cancellation. From them, the target has been completely drowned by interference signal in the

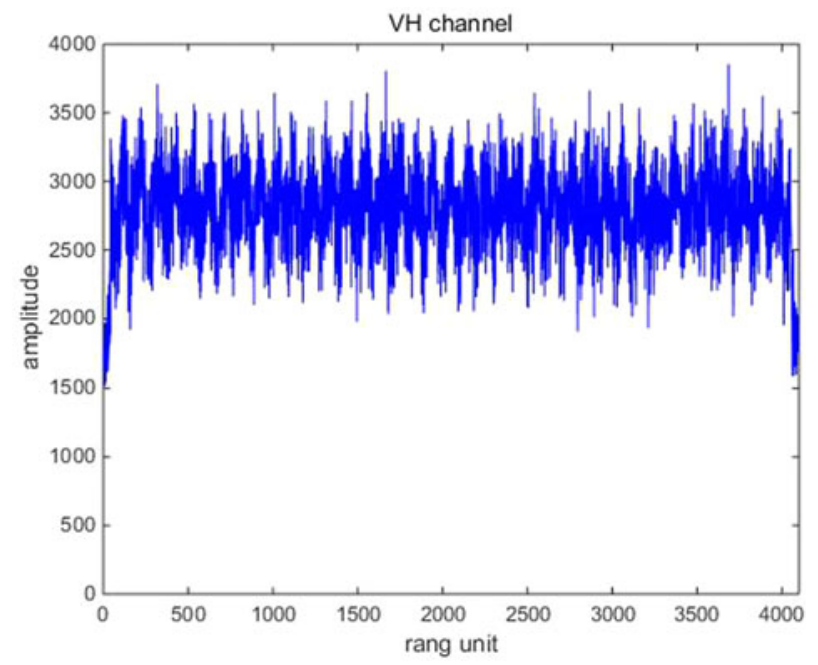

Fig. 5. Range profile of the $\mathrm{VH}$ channel for active blanket jamming.

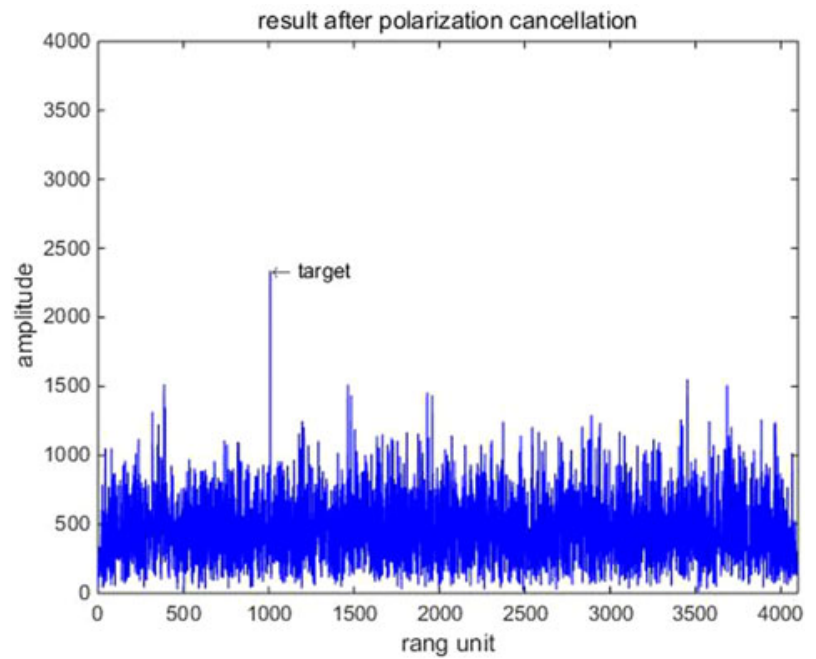

Fig. 6. Polarization cancellation result for active blanket jamming.

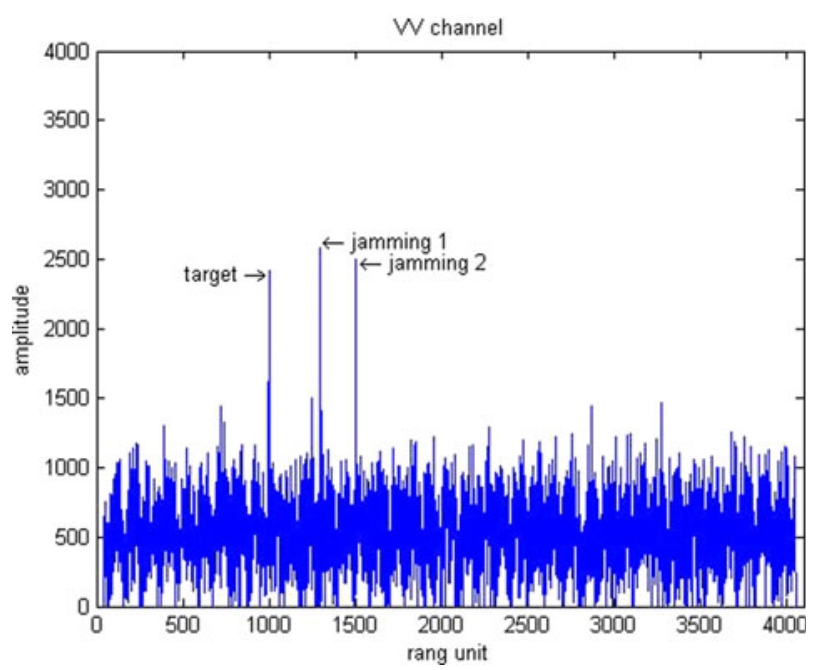

Fig. 7. Range profile of the VV channel for active deception jamming. 


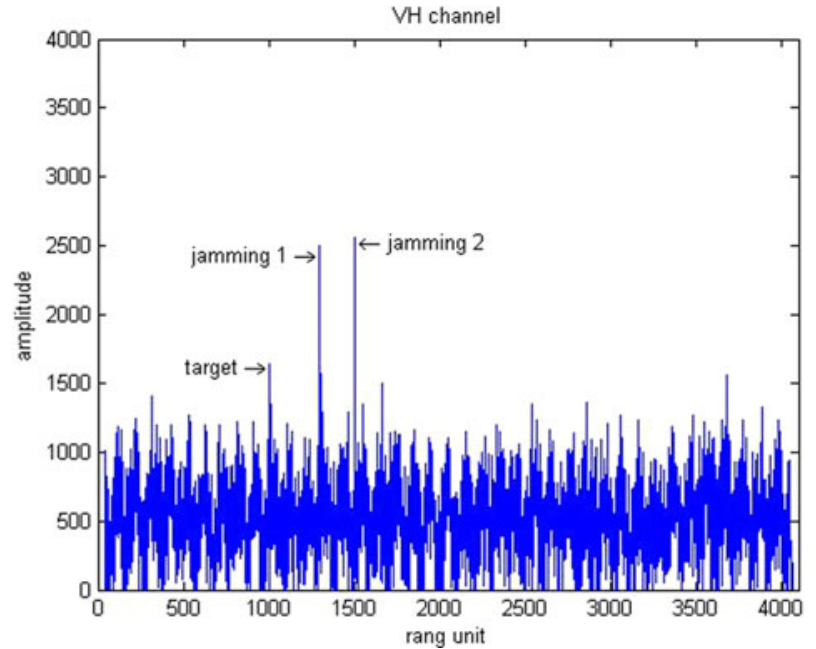

Fig. 8. Range profile of the VH channel for active deception jamming.

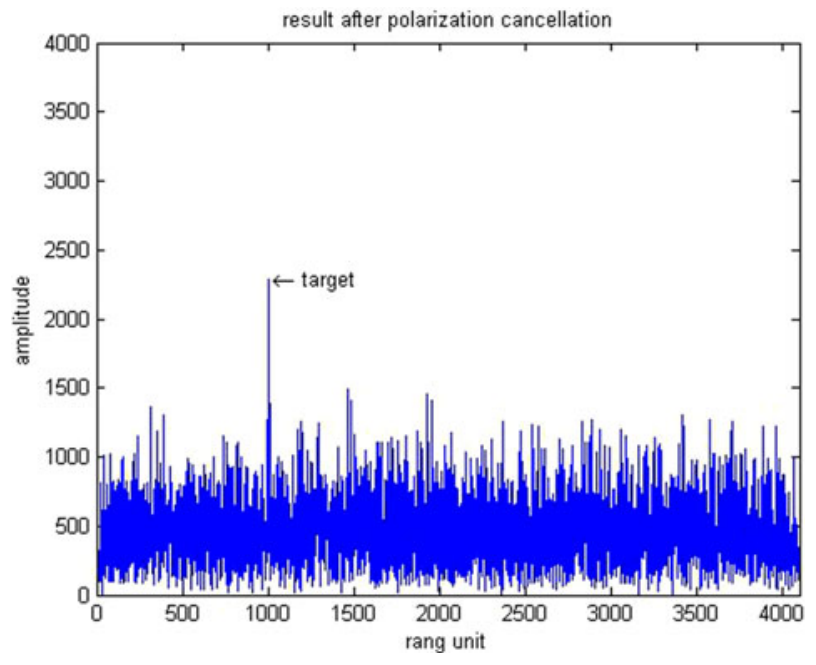

Fig. 9. Polarization cancellation result for active deception jamming.

Table 1. Comparison of monopulse angle measuring value.

\begin{tabular}{llll}
\hline & $\begin{array}{l}\text { Before polarization } \\
\text { cancellation }\end{array}$ & Method in [15] & $\begin{array}{c}\text { Method in } \\
\text { this paper }\end{array}$ \\
\hline $\begin{array}{l}\text { Target only } \\
\text { Mean }\end{array}$ & $-0.5030^{\circ}$ & 1 & 1 \\
$\quad \begin{array}{l}\text { Standard deviation } \\
\text { Target with active blanket jamming }\end{array}$ & $0.0601^{\circ}$ & 1 & 1 \\
$\quad$ Mean & $0.9673^{\circ}$ & $0.1020^{\circ}$ & $-0.3056^{\circ}$ \\
$\quad$ Standard deviation & $1.6789^{\circ}$ & $0.5627^{\circ}$ & $0.2714^{\circ}$ \\
Target with active deception jamming & $-0.3918^{\circ}$ & $-0.3265^{\circ}$ & $-0.4121^{\circ}$ \\
$\quad \begin{array}{l}\text { Mean } \\
\text { Standard deviation }\end{array}$ & $0.1735^{\circ}$ & $0.2481^{\circ}$ & $0.1279^{\circ}$ \\
\hline
\end{tabular}

VV channel, but the interference signal is perfectly eliminated and the target is effectively retained after polarization cancellation. Figures 7-9 are the same meaning as above for active deception jamming. Although they are difficult to distinguish from the real target in the VV channel, the deception decoys are also completely eliminated with the retaining of the real target after polarization cancellation.

In addition, the comparison of monopulse angle measuring value is given in Table 1 . In the experiment, the target and the active deception jammer are located at the same azimuthal direction of $-0.5^{\circ}$ off the boresight of the radar, while the active blanket jammer is nearly located at $1.5^{\circ}$. Making the angle estimation value acquired by the monopulse method without jamming as the standard value of the real target angle, it is obviously that the method proposed in this paper for countering active jamming can improve the angle-measuring accuracy greatly; and the result is also much better than the method in [15].

It is important to note that, the projection effect of geometry on the polarization vector is neglected in this paper, but the experimental results show that the proposed method is also quite meaningful.

\section{CONCLUSION}

In this paper, a countering method for active jamming is proposed based on dual-polarization radar seeker. Both active blanket jamming and active deception jamming can be eliminated perfectly, and the angle-measuring accuracy of monopulse radars is also improved greatly. Since it needs not any other hardware source more, the proposed method is in the vantage of size and complexity, which is significant for guidance radars. In addition, it can be realized before pulse compression, and does not affect the subsequent signal processing, such as coherent integration and imaging, so it is of a high value for engineering applications.

\section{ACKNDWLEDGEMENT}

This work was supported by the National Natural Science Foundation of China (Grant No. U1433126).

\section{REFERENCES}

[1] Gong, S.-x. et al.: Mathematic principle of active jamming against wideband LFM radar. J. Syst. Eng. Electron., 26 (1) (2015), 50-56.

[2] Dai, H.-y.; Wang, X.-s.; Li, Y.-z.: Novel discrimination method of digital deceptive jamming in mono-pulse radar. J. Syst. Eng. Electron., 22 (6) (2011), 910-916.

[3] Hurtado, M.; Xiao, J.-J.; Nehorai, A.: Target estimation, detection, and tracking, 'A look at adaptive polarimetric design'. IEEE Signal Process. Mag., 1 (2009), 42-50. 
[4] Qiao, X.-l. et al.: Anti-millimeter wave polarization agile active jamming, in Int. Conf. Microwave and Millimeter Wave Technology, Nanjing, 2007, 1-4.

[5] Tao, J. et al.: Anti-full polarization active jamming, in Second IEEE Conf. Industrial Electronics and Applications, Harbin, 2007, 2718-2722.

[6] Tao, J.-w.: Performance analysis for interference and noise canceller based on hypercomplex and spatiotemporal-polarisation processes. IET Radar, Sonar Navig., 7 (3) (2013), 277-286.

[7] Poelman, A.J.: Virtual polarisation adaptation-A method of increasing the detection capability of a radar system through polarisationvector processing. IEE Proc. F: Commun. Radar Signal Process., 128 (1981), 261-270.

[8] Song, L.-z. et al.: Scheme of adaptive polarization filtering based on Kalman model. J. Syst. Eng. Electron., 17 (1) (2006), 13-18.

[9] Wang, X.-s. et al.: Band characteristics of SINR polarization filter. IEEE Trans. Antennas Propag., 55 (4) (2007), 1148-1154.

[10] Mao, X.-p.; Liu, Y.-t.: Null phase-shift polarization filtering for highfrequency radar. IEEE Trans. Aerosp. Electron. Syst., 43 (4) (2007), 1397-1407.

[11] Dai, H.-y.; Li, Y.-z.; Liu, Y.: Spatial null phase-shift interference suppression polarization filter design for single polarized radars. Syst. Eng. Electron., 33 (2) (2011), 290-295.

[12] Jungsuek, O.; Kamal, S.: Compact, low profile, common aperture polarization, and pattern diversity antennas. IEEE Trans. Antennas Propag., 62 (2) (2014), 569-576.

[13] Park, P.K. et al.: Dual-polarization common aperture antenna for missile seekers includes multiple array, waveguide and stripline system, US Patent US2004056814-A1 and US6731241-B2, May, 2004.

[14] MBDA Missile Systems: Brimstone missile. http://brimstonemissile. com/brimstone/

[15] Varga, B.; DeWolf, G.: Polarization ECCM technique for missile radar systems, US Patent US5311192, 1994.

[16] Lee, J.-s.; Pottier, E.: Polarimetric Radar Imaging: from Basics to Applications, CRC Press, Boca Raton, FL, 2008.
[17] Dai, H.-y. et al.: Study on polarimetric measurement and radar target identification in instantaneous polarization radar system, in IET Int. Radar Conf., Guilin, 2009, 1-5.

[18] Barton, D.K.: Monopulse Radar, Artech House Publisher, Norwood, 1974 .

[19] Skolnik, M.: Radar Handbook, McGraw-Hill Professional, New York, 2008.

[20] Gu, J.-h.; Liang, G.; Yu, J.-p.: Channel calibration of amplitude-phase errors of DBF transmitter based on maximum likelihood estimation, in Int. Conf. Networks Security, Wireless Communications and Trusted Computing, Wuhan, 2009, 378-381

[21] Jeffrey, T.W.: Phased-Array Radar Design: Application of Radar Fundamentals, SciTech Publishing Inc., Chennai, 2008.

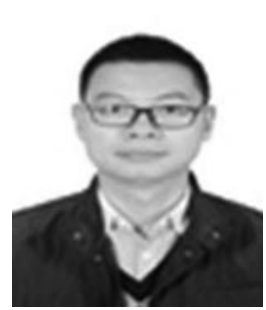

Qiang Zhang received his Ph.D. degree in Signal and Information Processing from the Chinese Academy of Sciences in 2012. He served as a senior engineer at Southwest China Institute of Electronic Technology in 2012-2015. He is now an Associate Professor at the College of Air Traffic Management, Civil Aviation Flight University of China. $\mathrm{He}$ is active in the areas of polarization technology, radar system design, image processing, and target recognition.

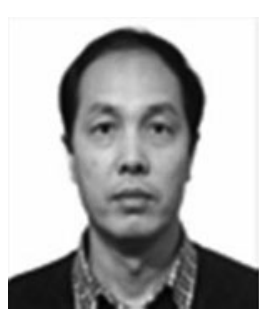

Weijun Pan received his Ph.D. degree in Computer Application from Sichuan University, China, in 2012. He is now a professor at College of Air Traffic Management, Civil Aviation Flight University of China. His research interests include radar system, air traffic management, computer application, and safety engineering. 